\title{
Introduction: Evidence and Causality in the Sciences
}

\author{
Phyllis Illari · Federica Russo
}

Published online: 14 January 2014

(C) Springer Science+Business Media Dordrecht 2014

\section{Evidence and Causality}

Causality is a vibrant and thriving topic in philosophy of science. It is closely related to many other challenging scientific concepts, such as probability and mechanisms, which arise in many different scientific contexts, in different fields. For example, probability and mechanisms are relevant to both causal inference (finding out what causes what) and causal explanation (explaining how a cause produces its effect). They are also of interest to fields as diverse as astrophysics, biochemistry, biomedical and social sciences. At the same time, there has been an explosion of interest in evidence, most obviously in biomedical contexts with the rise of 'evidence-based medicine', but also elsewhere, such as in social science. What is evidence? How do we decide what our best sources of evidence are?

This topos examines the relation between causality and evidence in different scientific areas. This involves questions about the foundations of the sciences, e.g. what is evidence and how does it contribute to causal knowledge? But it also involves questions about specific applications, e.g. how should we best deal with the many problems of evidence given by expert witnesses in court; and questions about policy-making, e.g. what constitutes evidence of

P. Illari

Department of Science and Technology Studies,

University College London, London, UK

e-mail: phyllis.illari@ucl.a.uk

F. Russo (ه)

Dipartimento di Studi Umanistici, Università di Ferrara, Ferrara, Italy

e-mail: federica.russo@unife.it causation that is relevant to the design of socio-economic and public health policies?

These questions are all of immense current concern. Pressure on health systems from challenges such as ageing populations and the obesity epidemic, coupled with severe financial constraints on public policy, means governments are demanding answers with increasing urgency. The time is ripe for a thorough discussion on causality and evidence. The contributions collected here are but the starting point, and we hope they will inspire many other scholars to engage with these topics, from either a philosophical or scientific angle.

\section{Papers in This Topos}

The present collection spans many disciplines, including physics and engineering, medicine, the social sciences, and law. These articles address a variety of topics surrounding the problem of evidence in their respective fields of application.

Several papers examine evidence in medicine. In particular, they discuss the idea of hierarchies of evidence, which are attempts to rank better and worse methods for generating evidence in medicine. Subtleties are developed in the papers, but broadly such hierarchies tend to prioritise evidence gained from randomized controlled trials over evidence gained from observational studies.

Barbara Osimani, in 'Hunting side effects and explaining them: should we reverse evidence hierarchies upside down?', takes issue with evidence hierarchies, particularly their use in assessing 'unintended effects'. Osimani explores the possibility of 'reversing the hierarchies', namely of giving comparatively more weight to observational studies than RCTs for such a purpose. 
In 'Down with the hierarchies', Jacob Stegenga also expresses concerns with evidence hierarchies, but his arguments are even more radical than the previous contribution. Stegenga questions the overall plausibility and appropriateness of hierarchies and provides several arguments for such concerns.

In 'Assessing the integrity of clinical data: when is statistical evidence too good to be true?', Margaret MacDougall concentrates on the top layers of evidence hierarchies, that is with statistical methods. MacDougall warns us to beware of statistical evidence that looks 'too good', as it may hide fraud. She lays out several methods for hunting for fraud and for evaluating statistical evidence in medical trials.

In 'Mechanisms and the evidence hierarchy', Brendan Clarke, Donald Gillies, Phyllis Illari, Federica Russo, and Jon Williamson instead focus on the bottom layers of the hierarchies, especially with the use of mechanisms to establish causal claims. The authors explain what evidence of mechanism means, how it complements evidence of difference making, and mostly, how such evidence ought to be evaluated.

There are then papers on evidence in other areas, namely social science, linguistic research, and in the law.

Attilia Ruzzene, in 'Process tracing as an effective epistemic complement', discusses cases in social science methodology where evidence for causal relations is gathered via the method of 'process tracing'. Ruzzene presents a criterion to establish when the evidence produced by such method indeed constitutes admissible evidence, thus contributing to establishing causal knowledge.

Christina Behme, in 'Assessing direct and indirect evidence in linguistic research', addresses the question of what constitutes evidence in linguistic research. In particular, she investigates what evidence may or may not support two important views, i.e. conceptualism and rational realism.

In 'Causation in personal injury law: the case for a probabilistic approach', Chris Miller argues for a more widespread and conscientious use of probabilistic evidence in the law, and in particular in cases of negligence. Miller restricts the discussion to the UK legal setting and uses several recent cases to illustrate his claims.

The collection finishes with three papers dealing with causality in physics.

In 'The contextual character of causal evidence', Mauricio Suárez argues for the view that evidence for causal claims is contextual. Notably, he shows cases where the same piece of evidence supports a causal claim in one context but not in another. Suárez uses the manipulationist framework to explain this idea and sets out the conditions to generalise this 'contextual' character of evidence beyond Woodward's interventionist framework.
Mathias Frisch, in 'Causality with a human face', attempts an answer to the 'causal sceptic' in the philosophy of physics, who claim that there is no place for causality in fundamental physics - the most famous of which is perhaps Bertrand Russell. Frisch provides several arguments showing how evidence for causal relations is a constant concern for physicists and how their reasoning is-pace these sceptics_-permeated with causal reasoning.

Wolfgang Pietsch, in 'The nature of causal evidence in physics, engineering, and other deterministic sciences', draws attention to deterministic contexts and asks a question about what constrains evidence in such cases. Pietsch revisits eliminative induction, in the tradition of Bacon and Mill, to show that they provide methods to establish the (ir)relevance of boundary conditions and of the background context against which causal relations have to be established.

\section{Genesis of the Papers}

Many of the papers in this collection were first presented at the seventh conference of the series 'Causality in the Sciences' (CitS) (http://www.kent.ac.uk/secl/philosophy/jw/ cits.htm), which was titled 'Evidence and causality in the sciences' (http://www.kent.ac.uk/secl/philosophy/jw/2012/ ecits/). In the end, 26 submitted talks were given, and the conference was a great success, attracting 84 registered speakers and attendees from all over the world, and enabling real collaboration between philosophy and various sciences, including: agronomy and environmental studies, biology, communication, epidemiology and medicine, law, philosophy, psychology and neuroscience, social science, statistics and computer science, and telecommunications. This continued the interdisciplinary tradition of CitS conferences, where fruitful discussions are set up among philosophers and scientists from various backgrounds.

After the conference, we issued an open call for papers. All papers have been refereed by anonymous experts. We are extremely grateful to all speakers, participants, and referees for making the conference such an enjoyable event and for contributing to putting together this collection. We also thank our colleagues in the CitS Steering Committee-Isabelle Drouet, Bert Leuridan, Julian Reiss, Erik Weber, Jon Williamson-for their enthusiasm in carrying on the causality venture over the years. We are also grateful to the various funding bodies that made the conference possiblethe AHRC, Mind Association, BSPS, the Faculty of Humanities, School of European Culture and Languages, University of Kent, and Kent's Centre for Reasoning - and to Fabio Paglieri, editor in chief of Topoi, for having accepted our proposal for this topos, and to the editorial assistants at Springer for taking prompt action whenever we needed help. 